\title{
Erratum to: Assessment of alkali-silica reaction damage through quantification of concrete nonlinearity
}

\author{
Krzysztof J. Leśnicki • Jin-Yeon Kim • \\ Kimberly E. Kurtis • Laurence J. Jacobs
}

Published online: 22 January 2013

(C) RILEM 2013

\section{Erratum to: Materials and Structures DOI 10.1617/s11527-012-9942-y}

An error in this manuscript was not addressed prior to electronic publication. While this error does not contribute in any way to the measurements or interpretation of concrete nonlinearity described, we felt a duty to correct the error to avoid introducing additional ambiguity in the identification of aggregate reactivity through the interpretation of expansion derived through standard concrete prism test methods. In the Introduction, on the second page of the manuscript, the application of rounding for the $0.04 \%$ expansion limit used for ASTM C1293 and

The online version of the original article can be found under doi:10.1617/s11527-012-9942-y.

K. J. Leśnicki · L. J. Jacobs

G. W. Woodruff School of Mechanical Engineering,

Georgia Institute of Technology, Atlanta,

GA 30332-0405, USA

K. J. Leśnicki

General Electric Global Research, Niskayuna,

NY 12309, USA

J.-Y. Kim · K. E. Kurtis $(\square) \cdot$ L. J. Jacobs

School of Civil and Environmental Engineering, Georgia Institute of Technology, Atlanta, GA 30332-0355, USA

e-mail: kimberly.kurtis@ce.gatech.edu;

kkurtis@ce.gatech.edu the $0.040 \%$ expansion used for CSA A23.2-14A was explained incorrectly and should read instead:

For ASTM C 1293, at 1 year (2 years if supplementary cementitious materials, SCMs, are used), the limit is $0.04 \%$ length change. (While this could make $0.035 \%$ the effective limit on expansion accounting for rounding, this is not commonly done in practice.) The Canadian standard version of the test, CSA A23.214A, uses a limit of $0.040 \%$.

As a result, expansion for Mix 1, discussed in Sect. 4.1, would indicate reactivity only if rounding, as implied by, but not generally practiced by ASTM C 1293 (not CSA A23.2-14A, as originally stated), was used. These corrections do not affect the classification of the mixes by expansion, which was based upon the commonly practiced interpretation of the ASTM C 1293 limit, and are extraneous to the assessment using the nonlinear acoustic resonance impact spectroscopy (NIRAS) method presented. 